International Journal of Computer Networks \& Communications (IJCNC) Vol.4, No.4, July 2012

\title{
Study the Effect of Base Frequency on the Performance of WiMAX Network Carrying Voice
}

\author{
Bassam F.Gumaidah ${ }^{1}$, Hasan H.Soliman ${ }^{2}$ and M.Obayya ${ }^{3}$ \\ ${ }^{1,2,3}$ Dep. of Communication Engineering, Faculty of Engineering, \\ Mansoura University, Egypt. \\ E-mail:bassam512360@hotmail.com
}

\begin{abstract}
WiMAX is stand for Worldwide Interoperability for Microwave Access. (WiMAX) embodies the IEEE 802.16 family of standards that provide wireless broadband access to residential and commercial Internet subscribers.

This technology takes the first position among the technologies these days. WiMAX can offers or adds some qualifications to the wireless technologies like high data rate, long coverage area and offers several types Quality of service to the customers. The long coverage area of WiMAX coming from the high transmit power and from the architecture of the network which is similar to mobile network, where the WiMAX base station architecture, is similar to GSM base station, need tower and can be sectorized, etc.

One of the feature of WiMAX devises is the wide range of base frequency from 2 to $66 \mathrm{GHz}$, on both licensed and free licensed spectrum [1] .In this paper the effect of base frequency on the WiMAX network performance will be studied we concern on three famous frequency $(2.4,3.5$ and $5.8 \mathrm{GHz})$ [2], which most of the WiMAX devises work on them.

OPNET MODELER is a simulation program used to simulate the work of networks with different values of base frequency. We use the modeler to study network of 12 WiMAX workstation connect and call each other by one WiMAX base station during 300 second.

The results show that the higher the base frequency the higher the Signal to Noise Ratio, that lead to high throughput, low packet end to end delay, finally lead to high mean opinion score (MOS) which mean good quality of voice. In the same time the results show that increase in the coverage area will lead to drop in Signal to Noise Ratio and MOS which will effect negatively in the quality of voice.
\end{abstract}

\section{Index Terms}

OPNET, SIMULATION, VOICE OVER IP, WIMAX.

\section{INTRODUCTION}

W

iMAX is a new technology offers a lot of internet service like (VoIP, IPTV, and other service on the internet) this paper will focus on VoIP service to study how the base frequency of base station can improve the performance of WiMAX network in both coverage area or the quality of voice. There are a lot of works on this field, some of them for 
International Journal of Computer Networks \& Communications (IJCNC) Vol.4, No.4, July 2012

study and the other for improvement. For example in [3] the authors provide a study of the bandwidth and delay requirements of VoIP services in WiMAX networks. They provide an analysis of the effects of Robust Header Compression, Payload Header Suppression, Voice Activity Detection with Discontinuous Transmission and other bandwidth affecting factors. In [4] the authors presents a new form of Adaptive Modulation (AM), which has the ability to enhance the data rate of Mobile WiMAX OFDMA system especially at low SNR values. In [5] the authors discusses a network architecture which is able to support real time services using 802.16 networks as a backhaul, providing an Integrated QoS approach over the 802.16 network. They introduce the concept of backup channel used to receive real-time services during the reservation setup time, avoiding packet losses or delays in these services. They also present an algorithm for dynamic management of the backup channel, and performance results of this algorithm. In [6] the author study both WiMAX and Wi-Fi networks, he focused in the coverage area for both, where he used constant values for WiMAX and Wi-Fi parameters and study the coverage area. He concluded that the best way to get wide coverage area is to use WiMAX network as backhole for Wi-Fi network.

Our work will take other way to study WiMAX network performance- as this paper will focus on VoIP- where the performance of WiMAX at different value of base frequency is studied and analyzed, to determine the base frequency which is enough to reach wide coverage area with good quality of voice.

First part of this paper will be background of WiMAX network. The second part will be overview about VoIP and the parameters affect on it. The third part is OPNET modeler overview. The last part will be simulation and results.

\section{WIMAX (802.16E) BACKGROUND}

WiMAX is a broadband wireless access that supports both fixed and mobile internet access. It is based on IEEE 802.16 and has maximum data rate of $75 \mathrm{Mbits} / \mathrm{sec}$ under optimal conditions [7]. WiMAX range covers up to several kilometers. As a result it can be used for providing wireless broadband across to cities and countries. It can be used as an alternative last mile solution to cable and DSL. WiMAX uses orthogonal frequency-division multiplexing OFDM and scalable orthogonal frequency-division multiple access (SOFDMA) so on this paper we will do the simulation on the tow type of multiplexing to see the effect of each one. It also supports multiple antennas for better coverage and better power consumption. Medium access control (MAC) layer of WiMAX uses a scheduling algorithm for the initial entry of the subscriber stations (SS) into the network. Then the base station (BS) allocates an access slot to SS and other subscribers cannot use that slot. The scheduling algorithm is also used for controlling the bandwidth efficiency and quality of service (QoS) parameters by changing the time slot duration based on the SS's application needs. WiMAX uses $2.3 \mathrm{GHz}, 2.5 \mathrm{GHz}$ and 3.5 $\mathrm{GHz}$ licensed bands. Since 2007 WiMAX technology is included in the IMT-2000 set of standards. IMT-2000 standards are defined by the radio communication sector of the International Telecommunication Union (ITU-R). As a result any country that recognizes IMT2000 standards is able to use to use WiMAX equipments [8].

WiMAX is based on PHY and MAC layer of the OSI (opens system interconnection) references model (fig 1), The PHY Layer identify advanced techniques at both modulation and error connection filed [7]. For example, Adaptive modulation and coding (AMC) is a modulation technique allows the base station to change the modulation code from low order modulation code (i.e.QPSK ${ }^{1}$ ) to high order modulation code (i.e.64QAM ${ }^{2}$ ) ,depend on the 
International Journal of Computer Networks \& Communications (IJCNC) Vol.4, No.4, July 2012

distance between the base station and workstations, that allows it to automatically increase effective range, when necessary, at the cost of decreasing throughput. As Higher-order modulation provides high throughput at sub maximum range, whereas lower-order modulation provides lower throughput at higher range, from the same base station [7].

${ }^{1}$ QPSK: Quadrature phase shift keying

${ }^{2}$ QAM: Quadrature Amplitude modulation.

Hybrid Automatic Repeat Request (HARQ) and Fast Channel Feedback (CQICH) are error correction techniques which are introduced with Mobile WiMAX to enhance coverage and capacity [7].

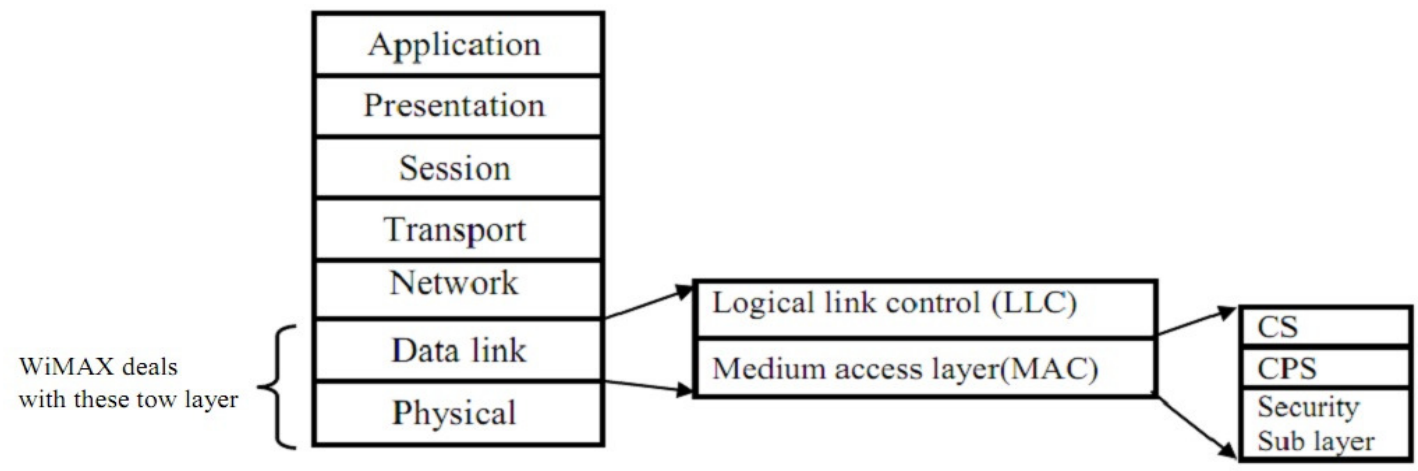

Figure 1 : OSI Reference model [7].

Table 1 summarizes the coding and modulation schemes supported in the Mobile WiMAX profile the optional UL codes and modulation are shown in italics [9], [10].

Table 1: Supported Code and Modulations [9]

\begin{tabular}{|c|c|c|c|}
\hline \multicolumn{2}{|c|}{} & DL & UL \\
\hline \multicolumn{2}{|c|}{ Modulation } & QPSK,16QAM,64QAM & QPSK,16QAM,64QAM \\
\hline \multirow{3}{*}{ Code Rate } & $\mathrm{CC}$ & $1 / 2,2 / 3,3 / 4,5 / 6$ & $1 / 2,2 / 3,5 / 6$ \\
\cline { 2 - 4 } & $\mathrm{CTC}$ & $1 / 2,2 / 3,3 / 4,5 / 6$ & $1 / 2,2 / 3,5 / 6$ \\
\cline { 2 - 4 } & repetition & $\mathrm{X} 2, \mathrm{X} 4, \mathrm{X} 6$ & $\mathrm{X} 2, \mathrm{X} 4, \mathrm{X} 6$ \\
\hline
\end{tabular}

The combinations of various modulations and code rates provide a fine resolution of data rates as shown in Table 2 which shows the data rates for 5 and $10 \mathrm{MHz}$ channels with PUSC sub-channels. The frame duration is 5 milliseconds. Each frame has 48 OFDM symbols, with 44 OFDM symbols available for data transmission. The highlighted values indicate data rates for optional 64QAM in the UL. 
International Journal of Computer Networks \& Communications (IJCNC) Vol.4, No.4, July 2012

Table 2 : Mobile WiMAX PHY Data Rates [9].

\begin{tabular}{|c|c|c|c|c|}
\hline parameter & downlink & uplink & downlink & uplink \\
\hline System bandwidth & \multicolumn{2}{|c|}{$5 \mathrm{Mhz}$} & \multicolumn{2}{c|}{$10 \mathrm{MHz}$} \\
\hline FFT size & \multicolumn{2}{|c|}{512} & 184 & 184 \\
\hline Null subcarrier & 92 & 104 & 120 & 280 \\
\hline Pilot subcarrier & 60 & 136 & 720 & 560 \\
\hline Data subcarrier & 360 & 272 & 30 & 35 \\
\hline sub channel & 15 & 17 & & \multicolumn{2}{c|}{1024} \\
\hline
\end{tabular}

\section{VOICE OVER IP}

Voice is analog and is converted to digital format before transmitting over Internet. This process is called encoding and the converse is called decoding and both are per-formed by voice Codecs. With bandwidth utilization becoming a huge concern, voice compression techniques are used to reduce bandwidth consumption. Voice compression by a codec adds an additional overhead of algorithmic delay. Thus, a codec is expected to provide good voice quality even after compression, with mini-mum delay.

The following table shows the bandwidth requirements of some common codecs. G.711 is the international standard for encoding telephone audio [11]. It has a fixed bit rate of 64kbps. G.723 and G.729 are low bit rate codecs at the expense of high codec complexity. G.723 is one of the most efficient codecs with the highest compression ratio and is used in videoconferencing applications [12]. G.729 is an industry standard with high bandwidth utilization for toll-quality voice calls [13]. G.726 uses ADPCM speech codec standard, and transmits at rates of 16, 24, 32, and $40 \mathrm{kbps}$. G.728 officially codes speech at $16 \mathrm{Kbit} / \mathrm{s}$ using low-delay code excited linear prediction .For example, during a call using G711 as codec, the amount of data transfer for both uplink and downlink will be $87.2 \times 2=174.4 \mathrm{Kbps}=0.1703$ Mbps $=10.21 \mathrm{Mb}$ per minute. So, G 711 uses $10.21 \mathrm{Mb} / \mathrm{min}$ per VoIP call where as G 729 uses $0.5 \mathrm{MB} / \mathrm{min}$ per voice call in the same way [14].

Table 3 : Voice codec [15].

\begin{tabular}{|c|c|c|c|}
\hline Codecs & Algorithm & $\begin{array}{c}\text { Bandwidth } \\
(\text { Kbps })\end{array}$ & $\begin{array}{c}\text { Ethernet Bandwidth } \\
\text { Usage (Kbps) }\end{array}$ \\
\hline G711 & PCM & 64 & 87.2 \\
\hline G729 & CS-ACELP & 8 & 31.2 \\
\hline G723.1 & Multi Rate Coder & 6.3 & 21.9 \\
\hline G723.1 & Multi Rate Coder & 5.3 & 20.8 \\
\hline G.726 & ADPCM & 32 & 52.2 \\
\hline G.728 & LD-CELP & 16 & 31.5 \\
\hline
\end{tabular}


International Journal of Computer Networks \& Communications (IJCNC) Vol.4, No.4, July 2012

\section{IV.OPNET MODELR}

There are several network simulation program such as Qualnet [16], NS2 (Network Simulation 2) [17], and Opnet modeler, each of them has its advantages and disadvantage. And all of them can be used to simulate wireless network, including WiMAX network and each program differs from the other, both in terms of the easily and the ability to use. OPNET MODELER was selected, as it has the following abilities:
a) Provides a comprehensive development environment supporting the modeling of communication networks and distributed systems.
b) Performing discrete event simulations.
c) Provides Graphical specification of model wherever possible; so models are entered via graphical editors.
d) Opnet modeler has library of models for most of the common networks around us.

OPNET is a research oriented network simulation tool. It is a very powerful software tool that simulates the real world behavior of wired and wireless networks. OPNET Modeler version 14.5 was used in this project for simulating WiMAX links. "The OPNET wireless module and the WLAN model provide high-fidelity modeling, simulation, and analysis of wireless networks, including the RF environment, interference, transmitter / receiver characteristics, and full protocol stack, including MAC, routing, higher layer protocols and applications. Furthermore, the ability to incorporate node mobility and interconnection with wire-line transport networks provide a rich and realistic modeling environment [18].

The OPNET WiMAX Specialized Model is available for OPNET Modeler® Wireless Suite and OPNET Modeler® Wireless Suite for Defense. It supports the IEEE 802.16-2004 and IEEE 802.16e-2005 standards [19].

\section{SIMULATION}

OPNET modeler 14.5 is used to simulate the WiMAX network in the following figure (fig.2), where we concern on some performance metric as follow:

1. Jitter: describes the degree of variability in packet arrivals. 75 milliseconds can tolerate (40 milliseconds) is preferred) of jitter delay [20].

2. Packet end to end delay: is the average time it takes for a packet to travel from its source to its destination. In consider to ITU recommendation G.114 The maximum amount of packet end to end delay that a voice call can tolerate one way is 150 milliseconds (100 milliseconds is preferred).

3. Throughput: is measure for the pure data which we can transfer successfully through the network.

4. Signal to noise ratio (SNR): is a measure of signal strength relative to background noise.

5. Path loss: is the reduction in power density (attenuation) of an electromagnetic wave as it propagates through space.

6. Mean Opinion Score (MOS): it provides a numerical indication of the perceived quality of received media after compression and/or transmission. It is expressed as a single number in a scale of 1 to 5 , where 1 is the lowest perceived quality, and 5 is the highest perceived quality [21]. 
International Journal of Computer Networks \& Communications (IJCNC) Vol.4, No.4, July 2012

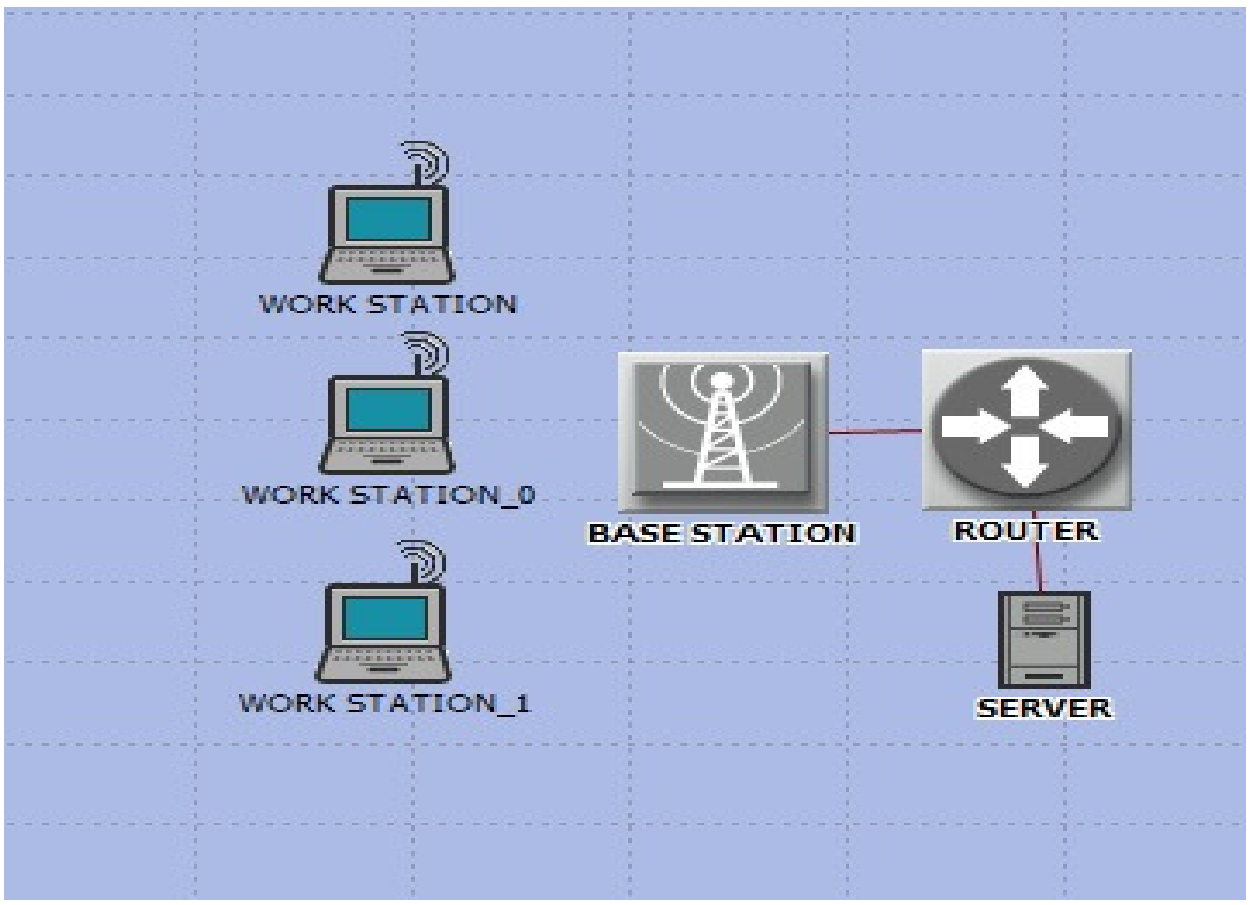

Figure 2 : network form.

Fig.2 is simple design of the studied WiMAX network, the simulation is implemented during 300 second, and the results will show the following:

1. Show the MOS (Mean Opinion Score), Packet End to End Delay, base station throughput, with G711, voice code.

2. Show how the path loss will influence by the Distance between the base station and the work stations, where it from $1 \mathrm{~km}$ to $54 \mathrm{~km}$ with base frequency $2.4,3.5$ and 5.8 $\mathrm{GHz}$ and channel Bandwidth $10 \mathrm{MHz}$ ).

3. Show how the SNR( signal to noise ratio) will influenced by the distance between the base station and the work stations, where it changes from $1 \mathrm{~km}$ to $54 \mathrm{~km}$ with base frequency $2.4,3.5$ and $5.8 \mathrm{GHz}$ and channel bandwidth $10 \mathrm{MHz}$.

The following table will resumes the network setting for all nodes:

1. Base station.

2. Workstation.

3. WiMAX configuration. 
International Journal of Computer Networks \& Communications (IJCNC) Vol.4, No.4, July 2012

Table 4: Network Setting .

\begin{tabular}{|c|c|c|}
\hline parameter & Base station & Work station \\
\hline Antenna gain $\mathrm{dB}$ & $15 \mathrm{~dB}$ & $-1 \mathrm{~dB}$ \\
\hline Transmitted power & $38 \mathrm{~W}$ & $0.5 \mathrm{~W}$ \\
\hline PHY profile & Wireless OFDMA $20 \mathrm{MHz}$ & Wireless OFDMA $20 \mathrm{MHz}$ \\
\hline PHY profile type & OFDM & OFDM \\
\hline \multicolumn{3}{|c|}{ Base station and Work station setting } \\
\hline & \multicolumn{2}{|c|}{ WiMAX attribute } \\
\hline Efficiency mode & \multicolumn{2}{|c|}{ Physical layer enable } \\
\hline Frame duration & \multicolumn{2}{|c|}{$5 \mathrm{msec}$} \\
\hline Symbol duration & \multicolumn{2}{|c|}{$102 \mathrm{msec}$} \\
\hline Number of subcarrier & \multicolumn{2}{|c|}{2048} \\
\hline Duplexing technique & \multicolumn{2}{|c|}{ TDD } \\
\hline Base frequency & \multicolumn{2}{|c|}{$2.4,3.5,5.8 \mathrm{GHz}$} \\
\hline Channel bandwidth & \multicolumn{2}{|c|}{$10 \mathrm{MHz}$} \\
\hline \multicolumn{3}{|c|}{ WiMAX configuration } \\
\hline
\end{tabular}

\section{RESULTS AND DISCUSSION}

OPNET modeler can give us the result as graphs, this graphs form a relation between the studied metric and simulation times or other effected factor like number of users. Also these results can be converted to excel data sheet to deal with them.

The simulation duration is 300 second and the scenario is repeated with change the distance from 1 to $54 \mathrm{~km}$ with each base frequency.

The following figure shows how we can change the base frequency in OPNET modeler: 
International Journal of Computer Networks \& Communications (IJCNC) Vol.4, No.4, July 2012

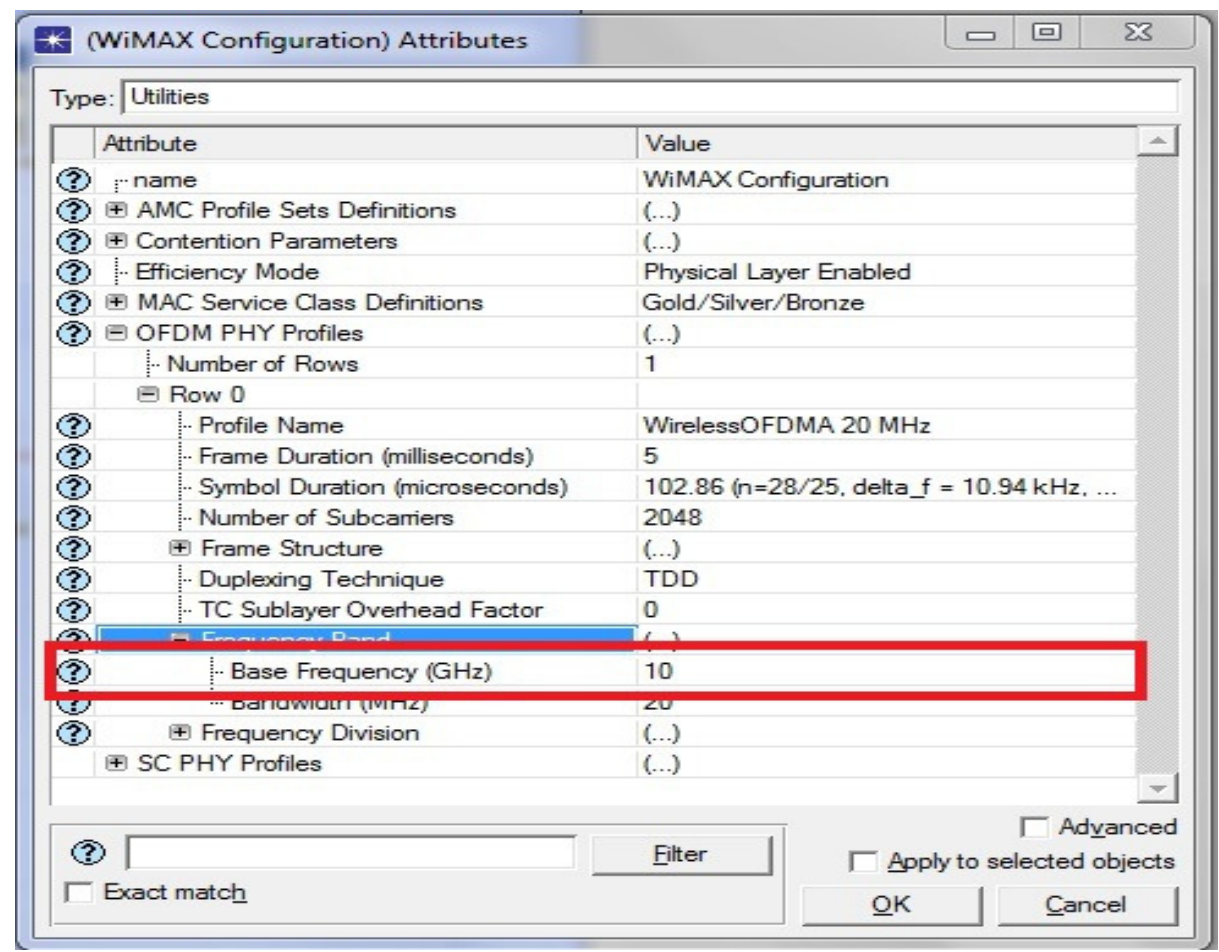

Figure 3 : base frquency setting.

First: Show the MOS (Mean Opinion Score), Packet End to End Delay, base station throughput, with G711 voice code:

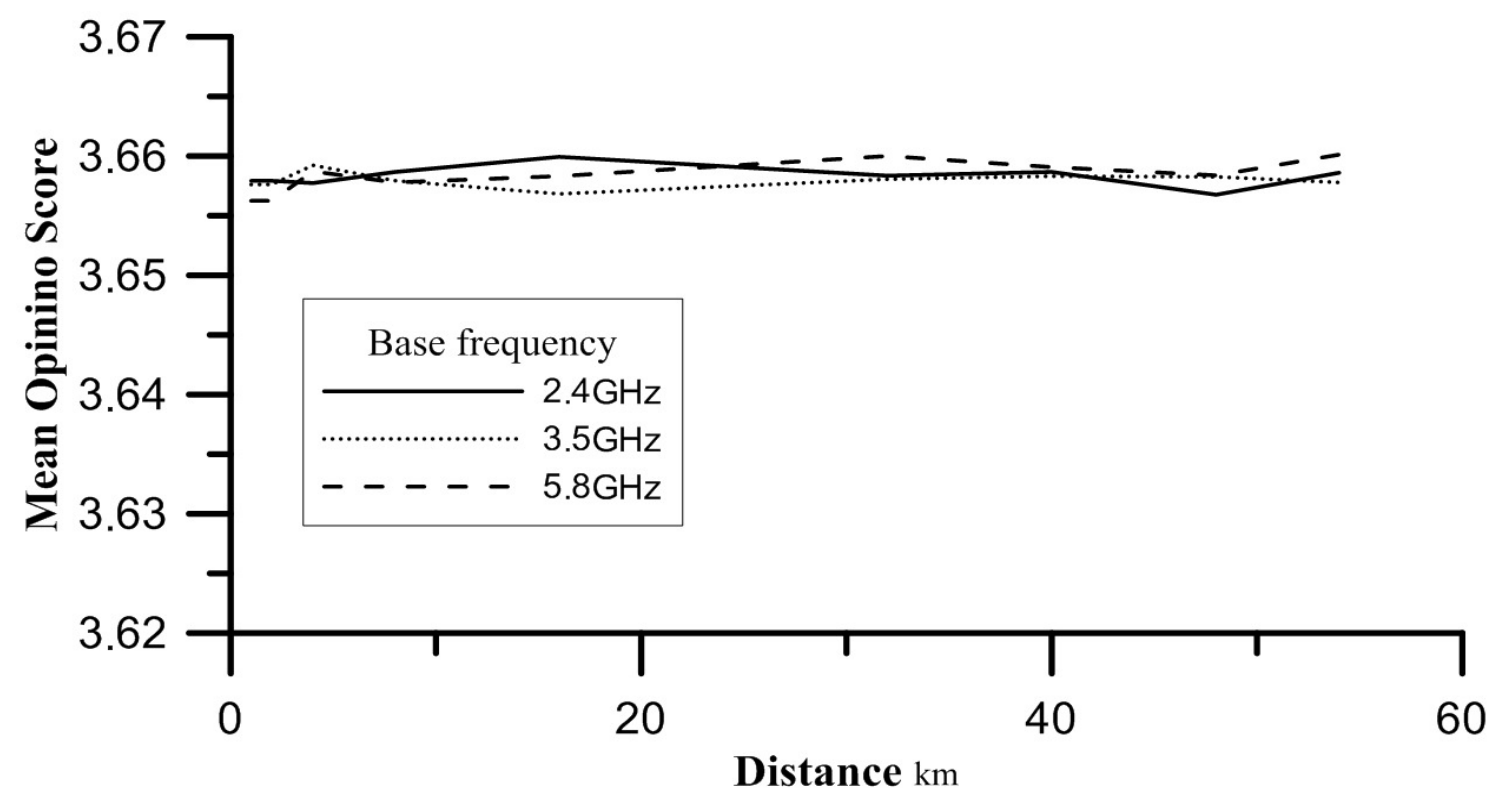

Figure 4: MOS. 
International Journal of Computer Networks \& Communications (IJCNC) Vol.4, No.4, July 2012

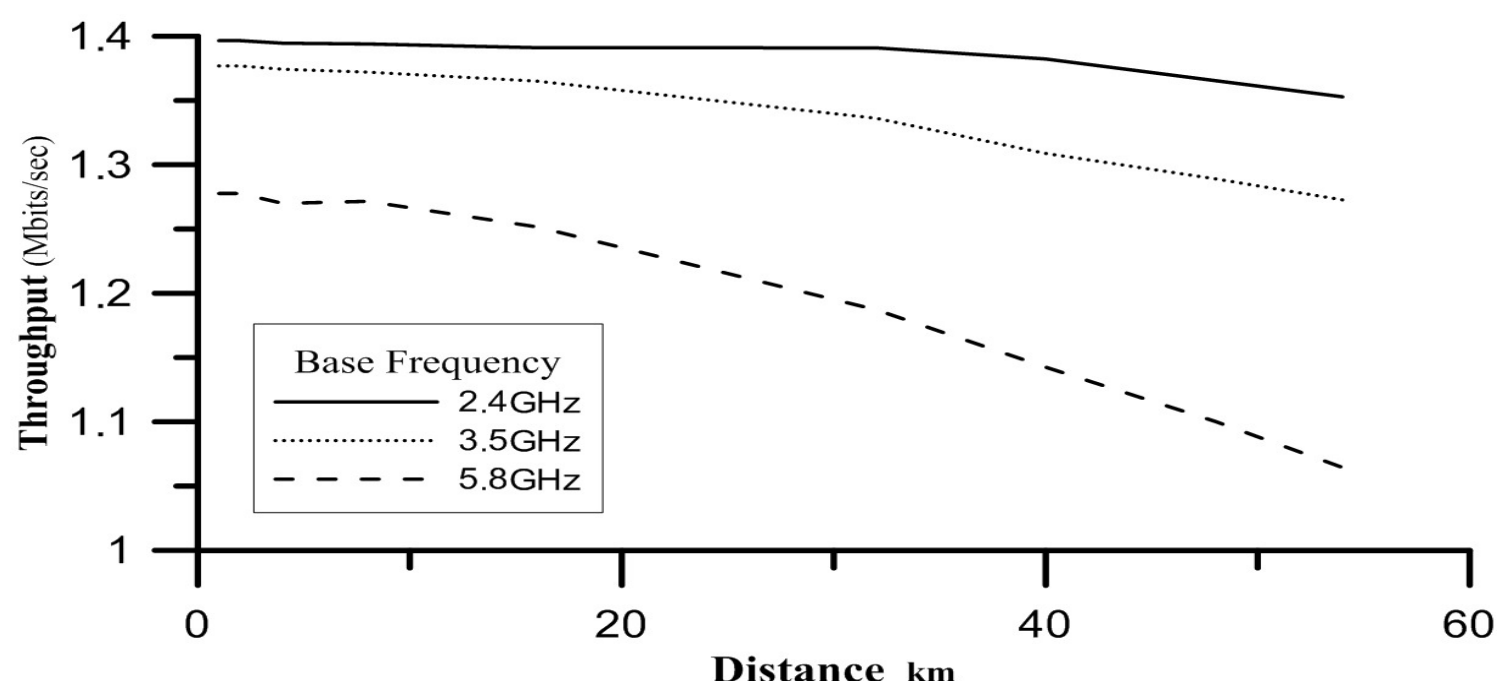

Figure 4 : throughput .

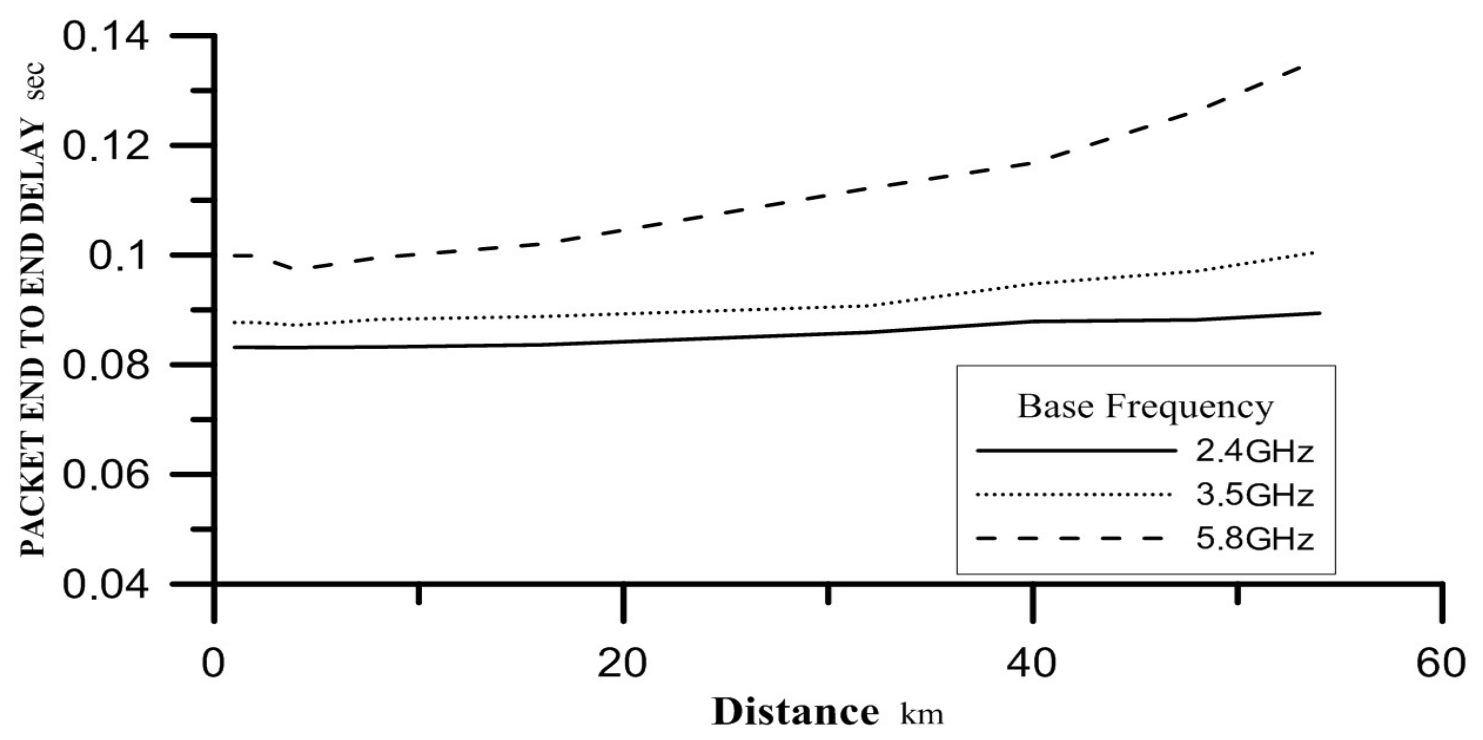

Figure 5 : packet end to end delay.

\section{Discussion:}

Fig.4 shows the mean opinion score with all used base frequency and no big different among them, as we only test 12 workstation so the effect not appars clearly. Fig.5 shows that the throughput go down with the distance increasing between work stations and base station with each base frequency because the attenuation that happened to signals, but what we notice that the lower base frequency $2.4 \mathrm{GHz}$ has the maximum throughput at any distance. Fig.6 show that packet end to end delay goes up as the distance increases between workstations and base station, also it is noticed that packet end to end delay increases sharply with high base frequency to unacceptable value, but with lower frequency increases slowly. We can explain 
International Journal of Computer Networks \& Communications (IJCNC) Vol.4, No.4, July 2012

these results as follow: when the base frequency decrease from 5.8 to $2.4 \mathrm{GHz}$ the signal is more able to diffract and penetrate through the obstacle and that will explains the high SNR and low Path Loss for lower frequency as we will see it next.

Second: Show how the path loss will influence by the Distance between the base station and the work stations, where it from $1 \mathrm{~km}$ to $54 \mathrm{~km}$ with base frequency $2.4,3.5$ and 5.8 $\mathrm{GHz}$ and channel Bandwidth $10 \mathrm{MHz}$.

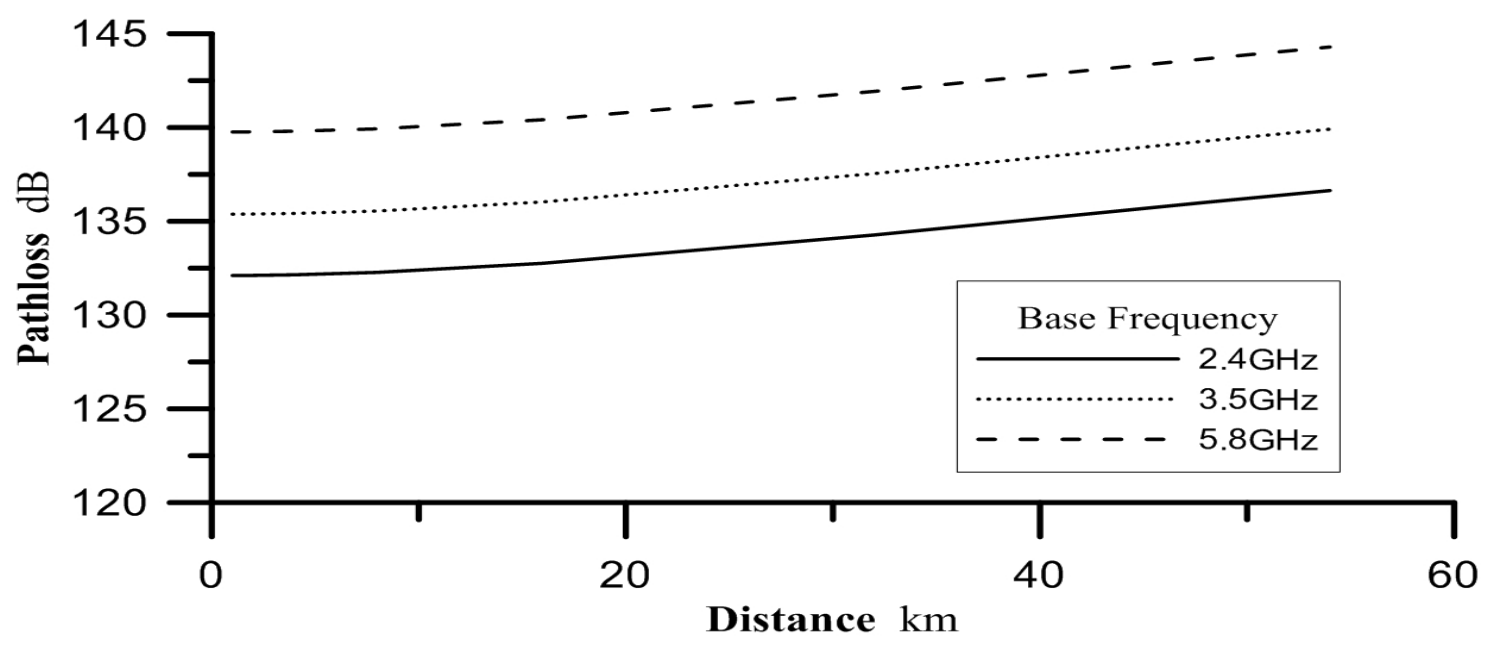

Figure 6 : path loss.

Third : Show how the SNR( signal to noise ratio) will influenced by the distance between the base station and the work stations, where it changes from $1 \mathrm{~km}$ to $54 \mathrm{~km}$ with base frequency $2.4,3.5$ and $5.8 \mathrm{GHz}$ and channel bandwidth $10 \mathrm{MHz}$.

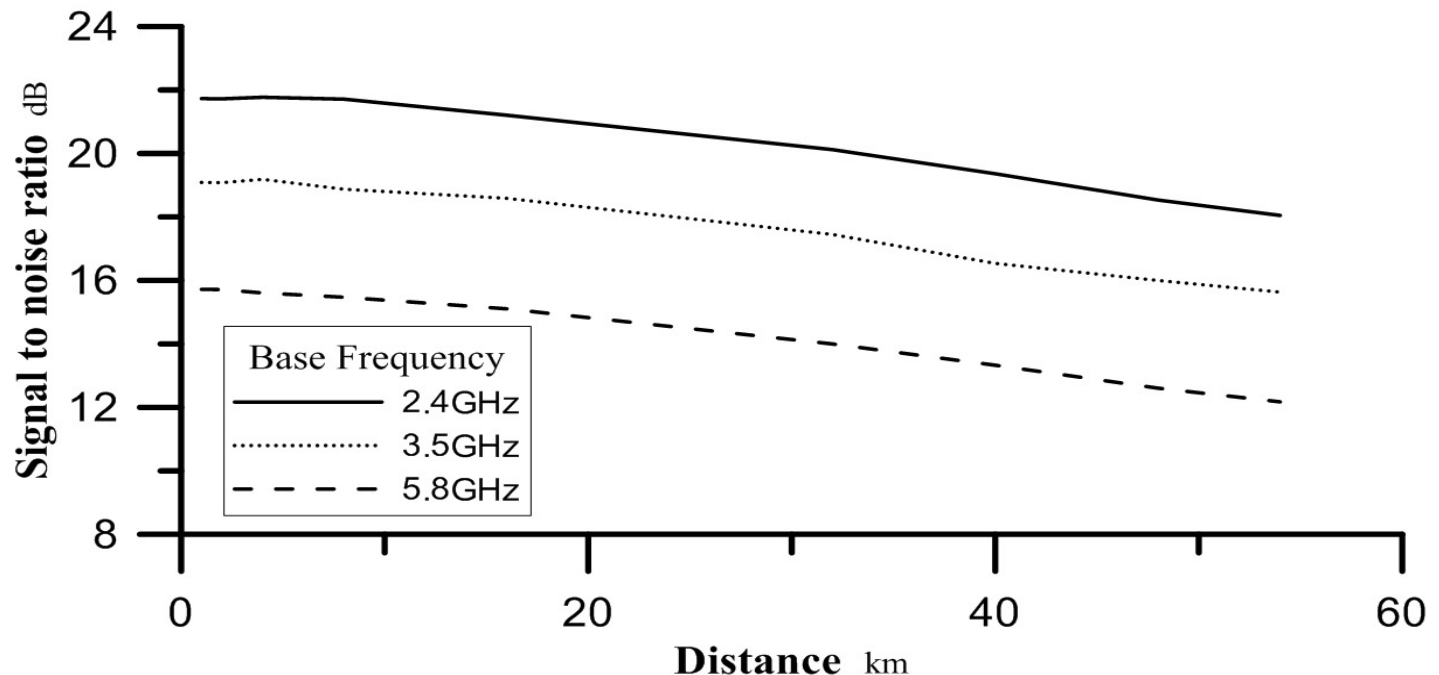

Figure 7:SNR. 
International Journal of Computer Networks \& Communications (IJCNC) Vol.4, No.4, July 2012

\section{CONCLUSION}

In the final of this paper it is concluded that the lower the base frequency the higher performance of WIMAX network .This a result, when the lowest base frequency $(2.4 \mathrm{GHz})$ is used the ability of signal to penetrate the wall diffract around the obstacle will be high ,in other mean the signal to noise ratio will be high, that lead to high throughput ,in turn high mean opinion score and low delay, and therefore good quality of voice .This ability of signals to penetrate and diffract goes down as the base frequency increase, that happened with the other base frequency $(3.5$ and $5.8 \mathrm{GHz})$ as the results show it. For long and wide coverage area it is recommend to use low base frequency for optimal performance of WiMAX network.

\section{VIII.References}

[1] Jeffrey G. Andrews, Arunabha Ghosh, and Rias Muhamed, Fundamentals of WiMAX: Understanding Broadband Wireless Networking.: Prentice Hall, 2007.

[2] WiMAX FORUM. WiMAX FORUM Spectrum database. [Online]. http://www.wimaxforum.org/resources/wimax-forum-spectrum-and-regulatory-database

[3] Ashraf A.Ali, Spyridon Vassilaras, and Konstantinos Ntagkounakis, "A Comparative Study of Bandwidth Requirements of VoIP Codecs Over WiMAX Access Networks," IEEE computer society, vol. 47, pp. 197-203, 2009.

[4] Ibrahim Ismail Al-kebsi, "Mobile WiMAX Performance Improvement Using a Novel Algorithm with a New Form of Adaptive Modulation," IJCSNS International Journal of Computer Science and Network Security, pp. 76-82, feb 2009.

[5] Miguel Almeida, Pedro Neves, Daniel Corujo, and Susana Sargento, "Improving the Experience of Real Time Services inWiMAX Networks," Instituto de Telecomunicações- Polo Aveiro, Universidade de Aveiro, Campus Universitário de Santiago, 2010.

[6] Azadeh Farzin, "OPNET Simulation of IEEE 802.11(Wi-Fi) and IEEE 802.16(WiMAX) in a small area," COMMUNICATION NETWORKS, Project ENSC 895-g200, spring 2010.

[7] Deepak Pareek, "WiMAX: Taking Wireless to the MAX," in WiMAX: Taking Wireless to the MAX.: Taylor \& Francis Group, LLC, 2006, pp. 149-189.

[8] Will Hrudey, "Streaming Video Content Over IEEE 802.16 / WiMAX Broadband Access," 2009.

[9] "Mobile WiMAX - Part I: A Technical Overview and Performance Evaluation," Aug 2006.

[10] Mohammad Tawhidul Alam Mohammad Saiful Islam, "WiMAX: An Analysis of the existing technology," 2009-01-21.

[11]ITU-T. Recommendation G.711. [Online]. http://www.itu.int/ rec/T-REC-G.711/e

[12] ITU-T. Recommendation G.723. [Online]. http://www.itu.int/ rec/T-REC-G.723/e

[13] ITU-T. Recommendation G.729. [Online]. http://www.itu.int/ rec/T-REC-G.729/e

[14] whirlpoo. [Online]. http://whirlpool.net.au/wiki/?tag=VoIP_Cod

[15] Cisco. Voice Over IP - Per Call Bandwidth Consumption. [Online]. http://www.cisco.com

[16] QualNet. [Online]. http://www.qualnet.com/content/products/qualnet

[17] (2011, may) The Network Simulator - ns-2. [Online]. http://www.isi.edu/nsnam/ns/index.html

[18] opnet. [Online]. http://www.opnet.com/support/des_model_library/WLAN80211.html 
International Journal of Computer Networks \& Communications (IJCNC) Vol.4, No.4, July 2012

[19] OPNET. [Online]. http://www.opnet.com/WiMAX/index.html

[20]F.P. Zhang, O.W.W. Yang, and B. Cheng, "Performance evaluation of jitter management algorithms," in Proc. of Canadian Conference on Electrical and Computer Engineering 2001, vol. 2, Toronto, May 2001, pp. 1011-1016.

[21] JERRY D.GIBSON and EDITOR, Multimedia Communications. Dallas ,Texas: Southern Methodist University, 2001. 